\title{
OLD AND NEW PROCESSES OF VORTON FORMATION
}

\section{Brandon Carter}

\section{D.A.R.C., Observatoire de Paris 92 Meudon, France}

Contribution to 35th Karpacs meeting (ed. J. Kowalski-Glikman)

Polanica, Poland, February 1999.

Astract After a brief explanation of the concept of a vorton, quantitative estimates of the vorton population that would be produced in various cosmic string scenarios are reviewed. Attention is drawn to previously unconsidered mechanisms that might give rise to much more prolific vorton formation that has been envisaged hitherto.

This review is an updated version of a previous very brief overview 1$]$ of the theory of vortons, meaning equilibrium states of cosmic string loops, and of the cosmological processes by which they can be produced in various scenarios. The main innovation here is to draw attention to the possibility of greatly enhanced vorton formation in cases for which the cosmic string current is of the strictly chiral type [2] that arises naturally in certain kinds of supersymmetric field theory.

It is rather generally accepted[3] that among the conceivable varieties of topological defects of the vacuum that might have been generated at early phase transitions, the vortex type defects describable on a macrosopic scale as cosmic strings are the kind that is most likely to actually occur - at least in the post inflationary epoch - because the other main categories, namely monopoles and walls, would produce a catastrophic cosmological mass excess. Even a single wall stretching accross a Hubble radius would by itself be too much, while in the case of monopoles it is their collective density that would be too high unless the relevant phase transition occurred at an energy far below that of the G.U.T. level, a possibility that is commonly neglected on the grounds that no monopole formation occurs in the usual models for the transitions in the relevant range, of which the most important is that of electroweak symmetry breaking.

The case of cosmic strings is different. One reason is that - although they are not produced in the standard electroweak model - strings are indeed produced at the electroweak level in many of the commonly considered (e.g. supersymmetric) alternative models. A more commonly quoted reason why the case of strings is different, even if they were formed at the G.U.T level, is that - while it may have an important effect in the short run as a seed for galaxy formation - such a string cannot be cosmologically dangerous just by itself, while a distribution of cosmic strings is also cosmologically harmless because (unlike "local" as opposed to "global" monopoles) they will ultimately radiate away all their energy and disappear. However while this latter consideration is indeed valid in the case of ordinary Goto-Nambu type strings, it was pointed out by Davis and Shellard 任 that it need not apply to "superconducting" current-carrying strings of the kind originally introduced by Witten [5]. This is because the occurrence of stable currents allows loops of string to be stabilized in states known as "vortons", so that they cease to radiate.

The way this happens is that the current, whether timelike or spacelike, breaks

the Lorentz invariance along the string worldsheet [6, 7, 8, 9], thereby leading to the possibility of rotation, with velocity $v$ say. The centrifugal effect of this rotation, may then 
compensate the string tension $T$ in such a way as to produce an equilibrium configuration, i.e. what is known as a vorton, in which

$$
T=v^{2} U
$$

where $U$ is the energy per unit length in the corotating rest frame 10, 11. Such a vorton state will be stable, at least classically, if it minimises the energy for given values of the pair of conserved quantities characterising the current in the loop, namely the phase winding number $N$ say, and the corresponding particle number $Z$ say, whose product determines the mass $M$ of the ensuing vorton state according to a rough order of magnitude formula of the form

$$
M \approx|N Z|^{1 / 2} m_{\mathrm{x}}
$$

where $m_{\mathrm{x}}$ is the relevant Kibble mass, whose square is the zero current limit value of both $T$ and $U$. If the current is electromagnetically coupled, with charge coupling constant $e$, then there will be a corresponding vorton charge $Q=Z e$.

Whereas the collective energy density of a distribution of non-conducting cosmic strings will decay in a similar manner to that of a radiation gas, in contrast for a distribution of relic vortons the energy density will scale like that of ordinary matter. Thus, depending on when and how efficiently they were formed, and on how stable they are in the long run, such vortons might eventually come to dominate the density of the universe. It has been rigorously established 12, 13, 14] that circular vorton configurations of this kind will commonly (though not always) be stable in the dynamic sense at the classical level, but very little is known so far about non-circular configurations or about the question of stability against quantum tunnelling effects, one of the difficulties being that the latter is likely to be sensitively model dependent.

In the earliest crude quantitative estimates 4 , 15] of the likely properties of a cosmological vorton distribution produced in this way, it was assumed not only that the Witten current was stable against leakage by tunnelling, but also that the mass scale $m_{\sigma}$ characterising the relevant carrier field was of the same order of magnitude as the Kibble mass scale $m_{x}$ characterising the string itself, which will normally be given approximately by the mass of the Higgs field responsible for the relevant vacuum symmetry breaking. The most significant development in the more detailed investigations carried out more recently [16, 17] was the extension to cases in which $m_{\sigma}$ is considerably smaller than $m_{x}$. A rather extreme example that immediately comes to mind is that for which $m_{x}$ is postulated to be at the G.U.T. level, while $m_{\sigma}$ is at the electroweak level in which case it was found that the resulting vorton density would be far too low to be cosmologically significant.

The simplest scenarios are those for which (unlike the example just quoted) the relation

$$
\sqrt{m_{\sigma}} \gtrsim m_{\mathrm{x}}
$$

is satisfied in dimensionless Planck units as a rough order of magnitude inequality. In this case the current condensation would have ocurred during the regime in which (as pointed out by Kibble[18] in the early years of cosmic string theory) the dynamics was dominated by friction damping. Under these circumstances, acording to the standard picture [3], the string distribution will consist of wiggles and loops of which the most numerous will be the shortest, characterised by a length scale $\xi$ say below which smaller scale structure will 
have been smoothed out by friction damping. The number density $n$ of these smallest and most numerous loops will be given by the (dimensionally obvious) formula

$$
n \approx \xi^{-3},
$$

in which the smoothing length scale $\xi$ itself is given by

$$
\xi \approx \sqrt{t \tau}
$$

where $\tau$ is the relevant friction damping timescale and $t$ is the cosmological time, which, using Planck units, will be expressible in terms of the cosmological temperature $\Theta$ by

$$
t \approx \Theta^{-2}
$$

in the radiation dominated epoch under consideration. According to the usual description of the friction dominated epoch [19, 3], the relevant damping timescale will be given by

$$
\tau \approx m_{\mathrm{x}}^{2} \Theta^{-3}
$$

from which it can be seen that the smoothing lengthscale $\xi$ that characterises the smallest and most numerous string loops will be given roughly by the well known formula

$$
\xi \approx m_{\mathrm{x}} \Theta^{-5 / 2} .
$$

At the time of condensation of the current carrier field on the strings, when the temperature reaches a value $\Theta \approx m_{\sigma}$, the corresponding thermal fluctuation wavelength $\lambda$ will be given by

$$
\lambda \approx m_{\sigma}^{-1} .
$$

Taken around the circumference, of order $\xi$, of a typical small string loop, the number of such fluctuation wavengths will be of order $\xi / \lambda$. In the cases considered previously [16, 17] it was assumed that the fluctuations would be randomly orientated and would therefore tend to cancel each other out so that, by the usual kind of random walk process the net particle and winding numbers taken around the loop as a whole would be expected to be of the order of the square root of this number of wavelengths, i.e. one would typically obtain

$$
N \approx Z \approx \sqrt{\xi / \lambda}
$$

However a new point to which I would like to draw attention here is that the random walk cancellation effect will not apply in case for which the current is of strictly chiral type so that the string dynamics is of the kind whose special integrability properties have recently been pointed out [2]. This case arises [5] when the string current is attributable to (necessarily uncharged) fermionic zero modes moving in an exlusively rightwards (or exclusively leftwards) direction. In such a case a loop possibility of cancellation between left moving and right moving fluctuations does not arise so that (as in the ordinary kind of diode rectifier circuit used for converting alternating current to direct curent) there is an effective filter ensuring that the fluctuations induced on the string will all have the same orientation. In such a case only one of the quantum numbers in the formula (2) will be independent, i.e. they will be restricted by a relation of the form $N=Z$, and their expected value will be of the order of the total number of fluctuation wavelengths round 
the loop (not just the square root thereof as in the random walk case). In such a strictly chiral case the formula (2) should therefore be evaluated using an estimate of the form

$$
N=Z \approx \xi / \lambda
$$

instead of (10)

Whereas even smaller loops will have been entirely destroyed by the friction damping process, those that are present at the time of the current condensation can survive as vortons, whose number density will be reduced in inverse proportion to the comoving volume, i.e. proportionally to $\Theta^{3}$, relative to the initial number density value given by (14) when $\Theta \approx m_{\sigma}$. Thus (assuming the current on each string is strictly conserved during the subsequent evolution) when the cosmological temperature has fallen to a lower value $\Theta \ll m_{\sigma}$, the expected number density $n$ of the vortons will be given as a constant fraction of the corresponding number density $\approx \Theta^{3}$ of black body photons by the rough order of magnitude formula

$$
\frac{n}{\Theta^{3}} \approx\left(\frac{\sqrt{m_{\sigma}}}{m_{x}}\right)^{3} m_{\sigma}^{3} .
$$

In the previously considered cases [16, 17], for which the random walk formula (10) applies, the typical value of the quantum numbers of vortons in the resulting population will be given very roughly by

$$
N \approx Z \approx m_{\mathrm{x}}^{1 / 2} m_{\sigma}^{-3 / 4},
$$

which by (2) implies a typical vorton mass given by

$$
M \approx\left(\frac{m_{x}}{\sqrt{m_{\sigma}}}\right)^{3 / 2}
$$

which, in view of (3), will never exceed the Planck mass. It follows in this case that, in order to avoid producing a cosmological mass excess, the value of $m_{\sigma}$ in this formula should not exceed a limit that works out to be of the order of $10^{-9}$, and the limit is even be smaller, $m_{\sigma} \ll 10^{-11}$, when the two scales $m_{\sigma}$ and $m_{x}$ are comparable.

The new point to which I wish to draw attention here is that for the strictly chiral case, as characterised by (11) instead of (10), the formula (2) for the vorton mass gives a typical value

$$
M \approx m_{\mathrm{x}}^{2} m_{\sigma}^{-3 / 2}
$$

which is greater than the what is given by the usual formula (14) by a factor $m_{\mathrm{x}}{ }^{1 / 2} m_{\sigma}^{-3 / 4}$. Although the vorton to photon number density ratio (12) will not be affected, the corresponding mass density $\rho=M n$ of the vorton distribution will be augmented by the same factor $m_{\mathrm{x}}^{1 / 2} m_{\sigma}^{-3 / 4}$. This augmentation factor will be expressible simply as $m_{\sigma}^{-1 / 4}$ when the two scales $m_{\sigma}$ and $m_{x}$ are comparable, in which case the requirement that a cosmological mass should be avoided leads to the rather severe limit $m_{\sigma} \lesssim 10^{-14}$. This mass limit works out to be of the order of a hundred $\mathrm{TeV}$, which is within the range that is commonly envisaged for the electroweak symmetry breaking transition.

The foregoing conclusion can be construed as meaning that if strictly chiral current carrying strings were formed (within the framework of some generalised, presumably supersymmetric, version of the Standard electroweak model) during the electroweak 
symmetry breaking phase transition, then the ensuing vorton population might conceivably constitute a significant fraction of the cosmological dark matter distribution in the universe. Although, according to (12), the number density of such chiral vortons would be rather low, their typical mass, as given according to (15) by $M \approx \sqrt{m_{\sigma}}$ would be rather large, about $10^{-7}$ in Planck units, which works out as about $10^{9} \mathrm{TeV}$.

An alternative kind of scenario that naturally comes to mind is that in which the cosmic strings themselves were formed at an energy scale $m_{\mathrm{x}}$ in the GUT range (of the order of $10^{-3}$ in Planck units) but in which the current did not condense on the string until the thermal energy scale had dropped to a value $m_{\sigma}$ that was nearer the electroweak value (below of $10^{-10}$ in Planck units). However since this very much lower condensation temperature would be outside the friction dominated range characterised by (3) would not be applicable. Preliminary evaluations of (relatively inefficient) vorton production that would arise from current condensation after the end of the friction dominated period are already available [17] for the usual random walk case, but analogous estimates for aumentation that might arise in the strictly chiral case have not yet been carried out. The reason why it is not so easy to evaluate the consequences of current condensation after the end of the friction dominated epoch (when radiation damping becomes the main dissipation mechanism) is that most of the loops present at the time of the current condensation would have been be too small to give vortons stable against quantum decay processes, a requirement which imposes a lower limit

$$
M \gtrsim m_{\mathrm{x}}^{2} / m_{\sigma}
$$

on the mass of a viable vorton. This condition is satisfied automatically by the masses estimated in the manner described above for vortons formed by condensation during the friction dominated era characterised by (3). On the other hand when (3) is not satisfied - in which case the lower limit (16) will evidently exceed the Planck mass - then the majority of loops present at the time of the carrier condensation phase transition at the temperature $\Theta \approx m_{\sigma}$ will not acquire the rather large quantum number values that would be needed to make them ultimately viable as vortons. It is not at all easy to obtain firmly conclusive estimates of the small fraction that will satisfy this viability condition. However it should not be too difficult to carry out an adaptation to the strictly chiral case of the kind of tentative provisional estimates (based on simplifying assumptions whose confirmation will require much future work) that have already been provided [17] for the generic of currennts built up by the usual random walk process.

The possibility of strictly chiral current formation is not the only mechanism whereby vorton formation might conceivably be augmented relative to what was predicted on the basis [17] of the previous estimates, which took no account of electromagnetic effects. There cannot be any electromagnetic coupling in the strictly chiral case [2], and in other cases where electromagnetic coupling will be typically be present it has been shown [20] that it will usually have only a minor perturbing effect on the vorton equilibrium states. However it has recently been remarked [21] that even though the averaged "direct" current that is relevant for vorton formation may be small, the local 'alternating' current can have a sufficiently large amplitude, $I$ say, for its interaction with the surrounding black body radiation plasma to provide the dominant friction damping mechanism, with a damping scale that instead of (7) will be given in rough order of magnitude by $\tau \approx m_{\mathrm{x}}^{2} I^{-1} \Theta^{-2}$. This means that instead of being restricted to the very early epoch when cosmological 
temperature was above Kibble limit value, i.e. when $\Theta \gtrsim \sqrt{m_{\mathrm{x}}}$, the period of friction domination can be extended indefinitely if the current amplitude satisfies $I \gtrsim m_{\mathrm{x}}^{2}$, a requirement that is easily compatible with Witten's [5] current saturation bound $I \lesssim e m_{\mathrm{x}}$ that applies (where the $e \simeq 1 / \sqrt{137}$ is the electromagnetic charge coupling constant) and in most cases even with the more severe limit $I \lesssim e m_{\sigma}$ that applies in cases for which instead of arising as a bosonic condensate, the current is due to femionic zero modes. Such a tendency to prolongation of friction dominance will presumably delay the decay of small scale loop structure and so may plausibly be expected to augment the efficiency of vorton formation in cases when $m_{\sigma}$ is below the limit given by (3), but a quantitative estimate of just how large this effect is likely to be will require a considerable amount of future work.

Despite the possibility that the effciency of vorton formation may have been underestimated by previous work, it still seems unlikely that vortons can constitute more that a small fraction of the missing matter in the universe. However this does not mean that vortons could not give rise to astrophysically interesting effects: in particular it has recently been suggested by Bonazzola and Peter 22] that they might account for otherwise inexplicable cosmic ray events.

The author is grateful to many colleagues, particularly Anne Davis and Patrick Peter, for helpful discussions on numerous occasions. 


\section{References}

[1] B. Carter, "Recent developments in Vorton Theory" Int. J. Theor. Phys. 36 24512458 (1997). astro-ph/9712116

[2] B. Carter, P. Peter, "Dynamics and integrability property of the chiral string model", preprint, Obs. Paris-Meudon hep-th/9905025

[3] E. P. S. Shellard \& A. Vilenkin, Cosmic strings and other topological defects, Cambridge University Press (1994).

[4] R. L. Davis, E. P. S. Shellard, Phys. Rev. D38, 4722 (1988); Nucl. Phys. B 323, 209 (1989);

[5] E. Witten, Nucl. Phys. B 249, 557 (1985).

[6] B. Carter, Phys. Lett. B 224, 61 (1989) and B 228, 466 (1989).

[7] B. Carter, in Formation and Interactions of Topological Defects (NATO ASI B349), ed R. Brandenberger \& A-C. Davis, pp 303-348 (Plenum, New York, 1995).

[8] P. Peter, Phys. Rev. D45, 1091 (1992).

[9] P. Peter, Phys. Rev. D46, 3335 (1992).

[10] B. Carter, Phys. Lett. 238, 166 (1990).

[11] B. Carter, P. Peter, A. Gangui, Phys Rev. D55, 4647 (1997) hep-ph/9609401.

[12] B. Carter, X. Martin, Ann. Phys. 227, 151 (1993).

[13] X. Martin, Phys. Rev. D50, 749 (1994).

[14] X. Martin, P. Peter, Phys. Rev D51, 4092 (1995).

[15] B. Carter, Ann. N.Y. Acad.Sci., 647, 758 (1991);

[16] B. Carter, Proc. XXXth Rencontres de Moriond, Villard-sur-Ollon, Switzerland, 1995, ed B. Guideroni, J. Tran Thanh Vân (Editions Frontières, Gif-sur-Yvette, 1995) hep-ph/9511225.

[17] R. Brandenberger, B. Carter, A-C. Davis \& M. Trodden, Phys.Rev. D54, pp 60596071 (1966) hep-ph/9605382.

[18] T. W. B. Kibble, J. Math. Phys. A 9, 1387 (1976); Phys. Rep. 67, 183 (1980).

[19] T. Vachaspati, A. Vilenkin, Phys Rev. D31, 3035, (1985).

[20] P. Peter, Phys. Lett. B298, 60 (1993).

[21] K. Dimopoulos \& A.-C. Davis, Phys. Rev. D57, 692 (1998). hep-ph/9705302)

[22] S. Bonazzola \& P. Peter, Astropart. Phys. 7, 161-122 (1997) hep-ph/9701246]. 